Gynäkol Rundsch 1990;30:I-VI

\title{
Contents, Vol. 30, 1990
}

Nr. 1 Originalarbeiten

Geburtshilfe bei HIV-positiven Schwangeren

Deslex, N.; Pavic, N.; Rudin, Ch.; Heinzl, S 1

Die Zystourethroskopie in der Diagnostik der Stressinkontinenz

Behr, J.; Winkler, M 8

Primärer Aldosteronismus in der Schwangerschaft

Casper, F.; Seufert, R.; Riedmiller, H.; Bauer, H 16

Hormonaler Synergismus, Reifung des Lungengerüstes und Surfactant Produktion in der

fetalen Schaflunge

Schellenberg, J.C.; Liggins, G.C 22

Hydrops fetalis bei Tachykardie: Diagnostisches und therapeutisches Vorgehen

Karrer, G.; Baumann, H.; Vetter, K.; Dudenhausen, J.; Huch, A.; Arbenz, U

Kurzfassungen von Zeitschriftenartikeln

Auffinden der bakteriellen Kolpitis beim Abstrich nach Papanicolaou

Platz-Christensen, J.-J.; Larsson, P.-G.; Sundström, E.; Bondeson, L

Plasmalevonorgestrelkonzentration und ovarielle Funktion bei Trägerinnen einer Levonorgestrel-freisetzenden Intrazervikalspirale

Ratsula, K.; Toivonen, J.; Lähteenmäki, P.; Luukkainen, T 41

Vergleich von fünf verschiedenen Clomiphenzitratdosierungen auf die Follikelbildung und deren Verteilung in den Ovarien beim Menschen

Shalev, J.; Goldenberg, M.; Kukia, E.; Lewinthal, D.; Tepper, R.; Mashiach, S.; Blankstein, $\mathrm{J} 43$

Erhöhte Werte von H-Y Antigen-positiven Lymphozyten bei Frauen mit Hirsutismus und die Wirkung einer Therapie mit Cyproteronacetat und Östradiol

Amice, V.; Bercovici, J.-P.; Nahoul, K.; Hatahet, M.-FL; Amice, J 45

Das Ovarialkarzinom vom Borderlinetyp

Chambers, J.T.; Merino, M.J.; Kohorn, E.F; Schwartz, P.E 47

Eine prospektive Studie Neuseelands über die Fertilität nach Entfernung von Kupfer-

Intrauterinpessaren wegen Kinderwunsch und wegen Komplikationen: Eine 4-Jahres-

Studie

Wilson, J.C 48

Mikrokürettage mit histopathologischer und zytologischer Beurteilung: Eine Alternative zur herkömmlichen Kürettage?

Löfgren, O.; Aim, P.; Ionescu, A.; Skjerris, J 50

Einñuss zweier oraler Kontrazeptiva auf Blutgerinnung und Fibrinolyse: eine Vergleichsstudie zwischen einer Dreiphasenpille mit Äthinylöstradiol und Levonorgestrel bzw.

Äthinylöstradiol und Gestoden

Omsjø, I.H.; Øian, P.; Maltau, J.M.; Østerud, B 52

Narbenhernien nach erneuter Laparotomie: ein übersehener Risikofaktor 
Lamont, P.M.; Ellis, H

53

Produktion von Prostaglandin E durch die Eihäute bei vorzeitiger Wehentätigkeit mit und ohne Chorioamnionitis

Lopez Bernal, A.; Hansell, D.J.; Khong, T.Y.; Keeling, J.W.; Turnbull, A.C

55

IV Inhaltsverzeichnis

Fortbildung

Die HIV-Infektion in der Geburtshilfe

Kosian, K.; Janisch, H 57

Varia

Empfehlungen des «Zürcher Gesprächskreises» zur oralen Kontrazeption

Keller, P.J 65

Veranstaltungskalender

67

Nr. 2 Originalarbeiten

Hemmung spontaner uteriner Kontraktionen durch Magnesium. Magnesiumtokolyse

Lechner, W.; Mayr, P.; Marth, C 69

Soziodemographische Charakteristika von Teilnehmern an Einführungskursen zur natür-

lichen Familienplanung. Endbericht einer Pilotstudie

Wolfram, G.; Widhalm, R 74

Chlamydieninfektion in der Gynäkologie

Carol, W.; Zenner, I.; Brunnemann, H.; Hartmann, M.; Salloum, L.; Alexander, H. . 84

Kurzfassungen von Zeitschriftenartíkeln

Vitamine als ätiologischer Faktor bei zervikalen Neoplasien - ein epidemiologischer Rück-

blick

Schneider, A.; Shah, K 92

Von der Einnahme oraler Kontrazeptiva abhängiges Mammakarzinom und Karzinom des weiblichen Genitaltraktes

James, J.; Schlesselman, Ph.D 95

Transvaginal gepulste Doppler-Flussmessungen des Corpus luteum von IVF-Patientinnen nach dem Embryotransfer

Baber, R.J. et al 98

Behandlung der Stressinkontinenz mittels Stamey-Operation bei älteren Frauen

Peattie, A.B.; Stanton, S.L 99

Die «einfache» Ovarialzyste: aspirieren oder operieren?

De Crespigny, L.Ch.; Robinson, H.P.; Davoren, R.A.M.; Fortune, D 101

Immunszintigraphie unter Verwendung von Ca-125-Antikörpern bei der Behandlung des

Ovarialkarzinoms

Brökelmann, J.; Bockisch, A.; Vogel, J.; Reinsberg, J.; Oèhr, P.; Biersack, H.J.; Krebs, D. 103

Fortbildung

Einführung zum Thema

Leodolter, S 106

12 Jahre Doppler-Sonographie in Gynäkologie und Geburtshilfe: Rückblick und Aus-

blick

Schneider, K.T.M.; Loos, W 108

Terminologie beim geburtshilflichen Doppler

Marhold, W.; Leodolter, S 111

Doppler-Ultraschalluntersuchungen in der Geburtshilfe 
Skodler, W 114

Gynäkologische Doppler-Flussmessungen

Schurz, B 119

Inhaltsverzeichnis $\mathrm{V}$

Neue Diagnostik der abnormen Uterusperfusion mittels transvaginaler Doppler-Strömungsmessungen in beiden Aa. uterinae

Rudelstorfer, R.; Deutinger, J.; Bernaschek, G 120

Wertigkeit der A/C-Ratio für die Voraussage der fetalen Aziditätssteigerung

Schaffer, H.; Lassmann, R.; Steiner, H.; Staudach, A 123

Diastolischer Flussverlust in Umbilikalarterie und fetaler Aorta; Korrelation zu Cardiotokographie und neonatalem Zustand

Lassmann, R.; Schaffer, H.; Steiner, H.; Staudach, A 126

Veranstaltungskalender 131

Ankündigung 105

Nr. 3 Originalarbeiten

Adjuvante Hormontherapie beim Endometriumkarzinom: Medroxyprogesteronazetat- versus

Tamoxifen-Medroxyprogesteronazetat-Sequenztherapie

Vavra, N.; Salzer, H.; Sevelda, P.; Breitenecker, G.; Czerwenka, K.; Kucera, H.; Österreichische Arbeitsgemeinschaft zur Therapie des Endometríumkarzinoms 133

Routinemässige Messung des grössten Fruchtwasserdepots im 3. Trimenon: Prognostische Bedeutung?

Haenel, A.F.; Fässli, C; Herrmann, U144

Örtlich ein- oder zweimalige Devitalisierung des Eileiters? Eine Untersuchung zur Methodik der bipolaren Hochfrequenzkoagulation

Yilmaztürk, A.; Schlüter, W 153

Pseudotumor cerebri in graviditate

Zeller, R.; Benz, J 161

Kurzfassungen von Zeitschriftenartikeln

HPV-16-DNS im Zervixkarzinom und dessen Lymphknoten: Ein Marker für frühe Meta-

stasierung?

Fuchs, P.G.; Girardi, F.; Pfister, H 166

Verwendung monoklonaler Antikörper gegen humane brusttumorassoziierte Antigene in der Diagnostik von Feinnadelaspirationen aus Knoten der Brust: Ergebnisse einer multizentrischen Studie

Natali, P.G.; Mottolese, M.; Perrone Donnorso, R.; Buffa, R.; Bussolati, G.; Goggi, G.;

Corradi, G.; Coscia-Porrazzi, L.; Ferretti, M.; Rondanelli, E.; Castagna, M.; Vecchione, A.; Siccardi, G.A 168

Erhöhte reduktive Aktivität der 17ß-Hydroxysteroiddehydrogenase durch Progestagene als mögliche Ursache für eine erhöhte Brustzellproliferation

Coldham, N.G.; James, V.H.T 170

Lipide, kardiovaskuläre Erkrankung und orale Kontrazeptiva: Eine praktische Übersicht

Upton, G.V 172

Therapieüberwachung durch Knochendichtemessung bei Östrogensubstitutionstherapie

Mazess, R.B.; Gallagher, J.C.; Notelovitz, M.; Schiff, I.; Utian, W 173

Medroxyprogesteronazetat und Psychotherapie in der Behandlung der pelvinen Kongestion: eine randomisierte kontrollierte Studie 
Farquhar, CM.; Robers, V.; Franks, S.; Pearce, S.; Wadsworth, J.; Beard, R.W. ... 175 1st die Kortisonprophylaxe bei Frühgeburten sinnvoll? Analyse von 12 kontrollierten Studien

Crowley, P.; Chalmers, J.; Keirse, M.J.N.C 177

VI

Inhaltsverzeichnis

Bestimmung des Blasenvolumens mittels Ultraschalls nach vorderer Kolporrhaphie

Paltieli, Y.; Degani, S.; Aharoni, A.; Shapiro, I.; Reiter, A.; Scharf, M.; Weiss, A. . 178

Bedeutung der Transvaginalsonographie in Geburtshilfe und Gynäkologie

Hata, K.; Hata, T.; Aoki, S.; Makihara, K.; Senoh, D.; Takamiya, O.; Kitao, M. ... 180

Bedeutung der gynäkologischen Ultraschalldiagnostik

Loutradis, D.; Antsaklis, A.; Creatsas, G.; Hatzakis, A.; Kanakas, N.; Gougoulakis, A.;

Michalas, S.; Aravantinos, D 182

Vergleich zwischen Diaphragma, Kondom und Rhythmusmethode als Kontrazeptivum

Dicker, D.; Wachsman, Y.; Feldberg, D.; Ashkenazi, J.; Yeshaya, A.; Goldman, J.A. . 183

Fortbildung

Vorzeitiger Blasensprung

Husslein, P 185

Vorzeitiger Blasensprung im 2. Trimenon

Fitz, R 188

Vaginale Noxen und Schwangerschaftsverlauf

Hosmann, J.W 191

Veranstaltungskalender

195

Nr. 4 Laudatio

Eduard Gitsch zum 70. Geburtstag

Reinold, E 201

Grusswort des Verlegers 205

Originalarbeiten

Operative Radikalität bei gynäkologischen Karzinomen

Burghardt, E 206

Die «Second-look»-Operation aus heutiger Sicht

Janisch, $\mathrm{H} \quad 214$

Die Senologie im Rahmen der Gynäkologie

Kubista, E 221

Wissen und Gewissen in der gynäkologischen Onkologie

Salzer, $\mathrm{H} \quad 228$

Einñuss einer verbesserten Diagnostik auf die Therapie

Husslein, P 233

Zur Ethik des Hochschullehrers

Huber, J.C 236

Subspezialisierung in der Gynäkologie - Pro und Kontra

Ludwig, $\mathrm{H} \quad 240$

Die Wahl des Operationsweges bei der Hysterektomie

Schwarz, R. 248

Geburtshilfe im Wandel - Rückblick und Ausblick

Wulf, K.-H 253 
Veranstaltungskalender

261

Autorenregister

263

Suppl. 1

Jahrestagung der Österreichischen Gesellschaft für Gynäkologie und Geburtshilfe 